
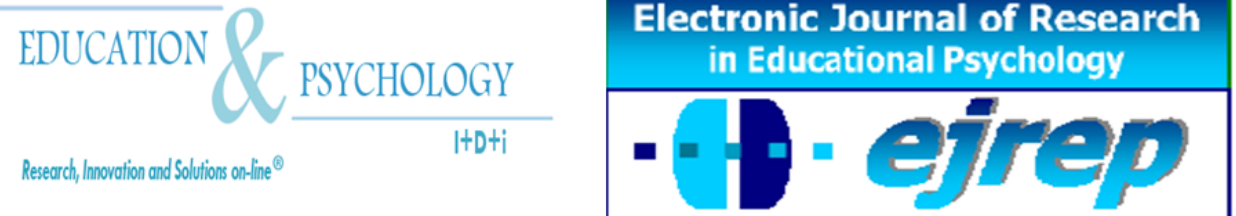

Editorial EOS

\title{
Aportaciones sobre la relación conceptual entre actitud y competencia, desde la teoría del cambio de actitudes
}

\section{Laura Arnau Sabatés ${ }^{1}$, Josep Montané Capdevila ${ }^{2}$}

${ }^{1}$ Profesora de Métodos de Investigación, Universidad Autónoma de Barcelona

${ }^{2}$ Catedrático de Orientación Educativa, Universidad Autónoma de Barcelona

\section{España}

Correspondencia: Laura Arnau. Universidad Autónoma de Barcelona. Campus de la UAB. Edificio G6. 08193 Bellaterra (Cerdanyola del Vallès). Barcelona. España. E-mail: laura.arnau@uab.cat

(C) Education y Psychology I+D +i and Editorial EOS (Spain) 


\title{
Resumen
}

En este trabajo se presenta una breve reflexión sobre las actitudes y competencias profesionales con el propósito de clarificar la complementariedad existente entre los dos constructos desde un punto de vista educativo, reconociendo la existencia de dos tendencias contrapuestas: la que identifica la actitud (y más concretamente, su dimensión afectiva) como una parte de la competencia y, la que asume que la actitud y la competencia son conceptos que funcionan en paralelo y que se implican mutuamente. El artículo se organiza en dos partes: una primera parte se centra en el concepto, estructura, modelos y desarrollo de las actitudes y su vinculación con las competencias profesionales y, una segunda, dónde se concreta el proceso de evaluación de ambos conceptos y se presentan algunas implicaciones prácticas para mejorar la intervención educativa.

Palabras Clave: actitud, cambio de actitud, competencia, dimensión cognitiva, dimensión afectiva, dimensión conductual.

Recibido: 10/05/10 Aceptación Inicial: 10/05/10 Aceptación Definitiva: 30/07/10

\begin{abstract}
In this work a brief reflection is presented about attitude and competence with the intention of clarifying their complementarity from an educational point of view, recognising two opposed tendencies: the one which identifies attitude (and more specifically, its affective dimension) as a part of the competence, and the other which assumes that attitudes and competencies are similar concepts that are mutually involved. The article is organized in two parts: the first one is focused on the concept, structure, models and development of the attitudes and its link with the competencies and, the second one, where the evaluation process of both concepts is specified and some practical implications to improve educative intervention are illustrated.
\end{abstract}

Keywords: attitude, change of attitude, competence, cognitive dimension, affective dimension, behavioral dimension.

Received: 05/10/10 Initial Acceptance: 05/10/10 Definitive Acceptance: 07/30/10 


\section{Introducción}

La lectura de los estudios sobre las competencias, analizados desde el modelo de cambio de actitudes, indican dos tendencias diferenciadas: a) las actitudes son sólo una parte de las competencias y, b) la competencia y la actitud son conceptos que podrían tratarse conjuntamente puesto que forman parte de un mismo proceso que se evalúa desde la acción o ejecución. Estas tendencias, además de comportar diferencias considerables entre sí, conllevan problemas conceptuales no resueltos y consecuencias en el modo de tratar las competencias.

La mayor parte de la literatura existente sobre competencias indica que la actitud es sólo una parte de la misma y, con frecuencia, se tiende a asimilar a los valores o emociones. Esta opción contradice el concepto clásico de la actitud. Si por el contrario, se considera que tanto la actitud como la competencia presentan un cierto paralelismo, se clarifican algunas dudas conceptuales puesto que ambos constructos integran, en su estructura, los tres factores que explican la conducta humana (cognitivo, afectivo y conductual) y los dos se evalúan desde su ejecución.

Desde un punto de vista educativo, parece importante clarificar la relación existente entre actitud y competencia. Puede resultar sumamente útil constatar hasta qué punto la actitud y la competencia funcionan como elementos casi paralelos y presentan más ventajas que la de considerar la actitud -especialmente su componente afectivo- sólo como una parte de la competencia. Así pues con esta reflexión pretendemos constatar hasta que punto la actitud y la competencia siguen procesos similares que se complementan entre sí.

\section{Aspectos generales de la actitud y su influencia en el comportamiento}

Las competencias adquieren cada vez más importancia para planificar la formación, evaluación, acreditación y ejecución de las profesiones, especialmente dentro de una sociedad basada en la información y en la cual existe la necesidad de orientaciones académicas holísticas que favorezcan el desarrollo del aprendizaje (Pérez Gómez, 2008). Son indicadores de dicha importancia el hecho que, tanto en España (Ley Orgánica 5/2002 de 19 de julio de las Cualificaciones y de la Formación Profesional) como en Europa (European Qualifications Framework for Lifelong Learning- EQF, 2008) se pretenda que las competencias permitan 
afrontar el Sistema de las Cualificaciones para la Acreditación Profesional (Echeverría, 2008). Sin embargo, el término competencia sigue siendo un constructo polisémico y en fase de desarrollo conceptual (Echeverría, 2008; Gimeno Sacristán 2008; Rodríguez Moreno, 2006; Tejada, 1999a, 1999b, 2005; Tejada y Navío, 2004). La mayoría de definiciones y estudios sobre competencias incluyen el concepto de actitud pero de manera diversa, lo cual favorece la dispersión y la confusión a la ya de por si compleja situación de la descripción de la competencia, en especial de los aspectos previos a su ejecución. Para entender mejor las posibles aportaciones de la actitud a la interpretación de la competencia se presentan algunas consideraciones preliminares.

\section{El origen de las actitudes y el cambio de actitudes}

En un principio la actitud o la tendencia a ejecutar una tarea, procedía de uno o dos factores: el factor cognitivo y, en algunas definiciones, se incluía el factor afectivo. Thurstone (1931) relacionó la actitud con el pensamiento y las emociones. Allport (1935), incluyó el componente conductual al definir la actitud como un aprendizaje que predispone a pensar, sentir y actuar de una manera determinada.

Katz y Stotland (1959), citado en Albarracín, Johnson, Zanna y Kumkale (2005) y, más recientemente, Arnau (2010), Bohner y Wänke (2002), Erwin (2001), Johnson, Maio y Smith-McLallen (2005) y Montané, Jariot y Rodríguez (2007), aceptan que las actitudes son predisposiciones a actuar de una determinada manera a partir de respuestas que podrían ser de carácter afectivo, cognitivo y conductual, siendo estos tres elementos, interrelacionados, los que configuran la actitud. Por su parte, Eagly y Chaiken $(1993,1998,2005,2007)$ reconocen la existencia de los factores afectivos, cognitivos y conductuales, no como constitutivos de la actitud - precepto asumido por la teoría triádica- sino como resultantes de una respuesta evaluativa global influenciada por la información que aporta cada uno de ellos a la tendencia a actuar, a veces por separado y, a veces, de manera interactiva. En este sentido, asumimos que la actitud es una tendencia a actuar de una manera determinada que se expresa en la evaluación a favor o en contra hacia un objeto y que está condicionada por la incidencia de los elementos cognitivos, afectivos y conductuales.

Algunas de las teorías más influyentes en el cambio de actitudes son las teorías de la consistencia y que parten de los trabajos de Heider $(1946,1958)$ sobre el modelo de equili- 
brio. Una de las más destacadas es la de la disonancia cognitiva (Festinger, 1957), que asume que las ideas predisponen a actuar y que la disonancia cognitiva tiende a unificar conceptos con comportamientos, según los intereses y la facilidad y/o dificultad de adaptar dichos comportamientos. Es posible que esta propuesta sólo se utilice como complemento para clarificar cómo el componente cognitivo tiende a influir en el comportamiento. Este concepto ha evolucionado y se acepta que la disonancia cognitiva tiende a unificar ideas con comportamientos, siempre que esté en juego un proceso de defensa del yo; Aronson $(1968,1999)$, demuestra que se produce la disonancia cuando está presente alguna otra variable de tendencia a la defensa del yo o de interés personal. Otros, la relacionan con los factores esperados y con la responsabilidad personal y la defensa de la autoestima (Cooper y Fazio 1984; Montané 1982, 1983, 2005). Sin embargo, Montané y colaboradores (2007), constataron la debilidad de las teorías de la consistencia, centradas exclusivamente en la defensa de la lógica y en la reducción de la disonancia, para lograr cambiar las actitudes.

Atendiendo a la concepción y descripción de la actitud hasta ahora expuesta sería poco fundamentado relacionar, de manera exclusiva, los componentes cognitivos o afectivos para explicar el cambio de actitudes, o utilizarlos, también, como elementos únicos para explicar el paso de la actitud a su ejecución, en forma de comportamiento y/o de competencia. Para poder iniciar un cambio de actitudes, es imprescindible considerar que hay que intervenir en las tres dimensiones de la actitud, tal y como señala el modelo de cambio de actitudes elaborado por Montané y colaboradores (2007). El modelo establece una secuenciación de cinco fases que condiciona tanto la elaboración de los contenidos de enseñanza-aprendizaje como la intervención pedagógica en el aula, a saber: evaluación inicial, mejora de la información, revisión de hábitos de comportamiento, integración de emociones y evaluación final. La eficacia de dicho modelo se ha constatado en los trabajos de Arnau (2010), Arnau y Montané (2010), Jariot y Montané (2009), Jariot y Rodríguez Parrón (2007), Montané (2010), Montané y Ferrer (1993), Montané y Jariot (2005), Montané y colaboradores (2007), Montané, Martínez y Ja$\operatorname{riot}(2004)$.

Características, desarrollo y ejecución de las actitudes

De las concepciones de actitud expuestas en las líneas precedentes, se desprenden tres grandes consideraciones que pueden ser de interés para relacionar la actitud con la competen- 
cia: la incidencia de los tres componentes (cognitivo, conductual y afectivo) en la ejecución de la actitud, su interacción y la reciprocidad de éstos con la actitud.

a) Los tres componentes influyen en la ejecución de la actitud directamente

La influencia de los tres factores: cognitivo, conductual y afectivo, en este triple flujo, explican en gran parte la predicción y ejecución del comportamiento deseado o esperado (Azjen, 2001; Ajzen y Fishbein, 2005; Fazio, 1995; Olson y Stone, 2005). Según esta afirmación, las ideas, los comportamientos y las emociones influyen directamente en la manera de actuar. Por consiguiente, de alguna u otra forma, si se mejora la información, las destrezas y hábitos de comportamiento y se introducen emociones y sentimientos favorables a la ejecución, se mejorarán los resultados de la actitud y la predición del comportamiento (Ajzen 2001; Ajzen y Fishbein, 2005; Johnson, Maio y Smith-McLallen, 2005; Maio y Haddock, 2010; Marsh y Wallace 2005; Montané et al., 2007). Dicha predicción se incrementa cuando las actitudes están basadas en la experiencia directa y dependen de la propia personalidad (Fazio y Zanna, 1981); son más accesibles a la memoria (Fazio, 1995; Gawronski y LeBel, 2008); son extremas (Holland, Verplanken y Knippenberg, 2002; Petersen y Dutton, 1975); son consistentes (Eagly y Chaiken, 2005) y no son ambivalentes (Armitage y Conner, 2000). El enfoque triádico es un hecho a considerar tanto en la estructura como en la intervención o formación de las actitudes (Eagly y Chaiken, 2007; Fabrigar, MacDonald y Wegener, 2005).

Resulta difícil reducir la actitud sólo a la predisposición. Es cierto que, académicamente, la actitud puede entenderse como predisposición a la ejecución, pero para poder constatar que existe una predisposición habrá que evaluar la ejecución de la actitud. Por consiguiente, la evaluación de la ejecución de la actitud forma parte intrínseca de la misma actitud. La observación de la conducta o las diferentes formas de evaluación de la actitud, son un ejemplo de cómo la amplia literatura relacionada con la evaluación de las actitudes indica que la ejecución de la actitud resulta necesaria para complementar el concepto de actitud (Albarracín et al., 2005; Bohner y Wänke, 2002; Erwin, 2001; Fazio y Olson, 2003; Gawronski, 2007; Haddock y Maio, 2004; Krosnick, Judd y Wittenbrink, 2005; Maio y Haddock, 2010). 
b) Las tres dimensiones interactúan entre sí

Las tres dimensiones de la actitud, además de incidir cada una por separado en la respuesta de la actitud, están íntimamente relacionadas entre sí. De este modo, los contenidos cognitivos influyen a su vez y, dependen, de los componentes afectivos y de los elementos conductuales en forma de hábitos de comportamiento y de destrezas. Son numerosos los autores que relacionan los procesos de pensamiento con las emociones y los hábitos, y consideran estos tres componentes los que preparan la ejecución de la actitud y, por extensión, del comportamiento si se realiza un paralelismo entre la predisposición interna y la ejecución de la actitud (Eagly y Chaiken, 2005, 2007; Maio y Haddock, 2010; Montané et al., 2007; Zanna y Rempel, 1988).

Esta nueva concepción de la estructura de la actitud dificulta que una sola dimensión en si misma, pueda explicar la predisposición a ejecutar la actitud. Según esto será aventurado reducir la actitud a una sola dimensión, por ejemplo a la afectiva. Hipotéticamente sólo seria posible conseguir un cambio de actitudes en el caso de que las restantes dimensiones (cognitiva y conductual) estuvieran presentes de manera implícita y presentaran una valencia y consistencia suficiente entre ellas (Maio y Haddock, 2010). De hecho, se define que la actitud es el resultado de una combinación de los tres factores (Albarracín et al., 2005; Bohner y Wänke 2002; Eagly y Chaiken, 1993, 2005, 2007; Erwin 2001; Montané et al., 2007).

c) La manifestación de la actitud influye a su vez en los mismos elementos que la ayudaron a emerger

La expresión de la actitud influye a su vez, de manera interactiva, en los mismos elementos que incidieron en su ejecución (Ajzen y Fishbein, 2005; Albarracín et al., 2005; Marsh y Wallace, 2005; Montané et al., 2007). Lógicamente si la conducta es el resultado de los tres componentes de la actitud, puesto que están íntimamente relacionados, ésta a su vez influye en los tres factores ya mencionados, aunque seguramente de manera muy diversa según el objeto o el resultado de la ejecución de la actitud (Clore y Schnall, 2005; Kruglanski y Stroebe, 2005; Olson y Stone, 2005). 
Se constata que las actitudes predisponen a ejecutar determinados comportamientos que, una vez puestos en práctica, pueden remodelar o cambiar las actitudes, llegando a desarrollar otras nuevas que requieran nuevos comportamientos y, por extensión, nuevas competencias. Actitud y comportamiento, siempre están en desarrollo, puesto que forman parte de un mismo proceso de intercomunicación y de interdependencia recíproca (Ajzen y Fishbein, 2005; Eagly y Chaiken, 1993, 2005, 2007; Fazio, 1995; Fazio y Zanna, 1981; Olson y Stone, 2005). La ejecución del role playing, es un ejemplo de cómo la expresión de la conducta puede transformar el mismo comportamiento anterior $\mathrm{y}$, en consecuencia, cambiar las actitudes previas (Jannis 1968, citado en Olson y Stone, 2005).

A modo de resumen, el siguiente esquema (figura 1) visualiza la triple interacción de los tres factores y su relación con la actitud así como su manifestación en forma de comportamiento y por extensión, de competencia, entendida como "saber actuar".

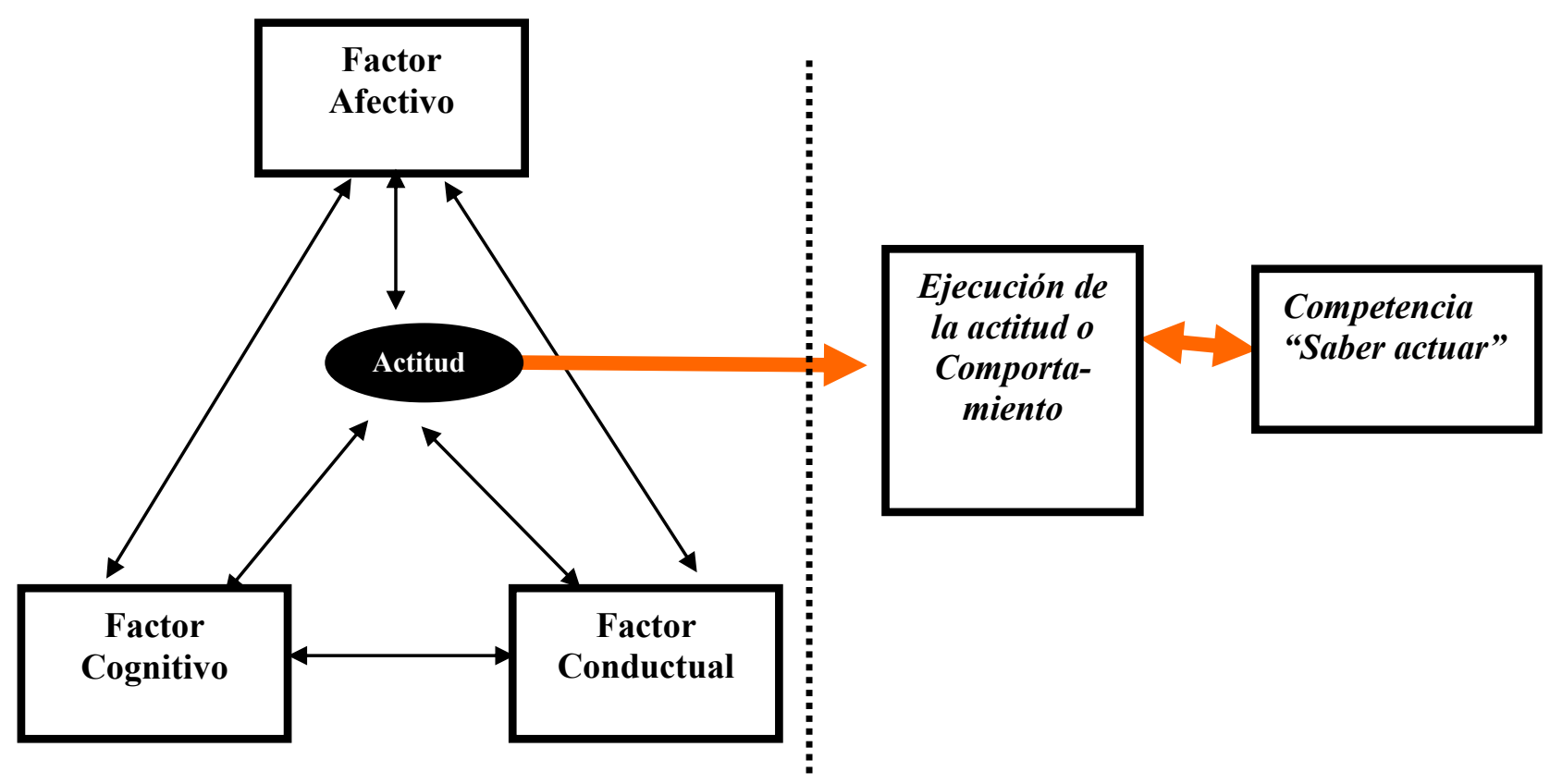

Figura 1. Relación de los tres factores de la actitud y su triple interacción.

La riqueza y complejidad de dicha interrelación ofrecen un marco de comprensión que proporciona pistas para intervenir y explicar no sólo los procesos de la actitud, sino todos los de la competencia que sean similares a los de la actitud. Además de todo lo dicho, no es de extrañar que surjan un conjunto de matices en los que se describe como, supuestamente, se 
relacionan los tres factores. Así, aspectos cognitivos relacionados con las emociones, se manifiestan en conceptos de persuasión y contundencia, según la teoría del comportamiento planificado de Ajzen y Fisbein (1980), otros relacionan entre si emociones y destrezas en términos de comportamiento (Erwin, 2001; Levitan y Visser, 2008).

Existen, también, otras dimensiones de la actitud que pueden ser útiles en relación con el comportamiento, a saber: la relación con el objeto negativa, neutra o positiva abre un intervalo de relación que condicionará la forma de evaluar el comportamiento dentro de un continuo de rechazo- atracción (Petersen y Dutton, 1975). Este continuo en forma de evidencia cierta o equivocada, destreza adecuada o inadecuada, sentimiento de rechazo o atracción, incide en las tres dimensiones. De esta forma se explican los diferentes matices con que puede presentarse la ejecución de la acción y su influencia en el desarrollo y manifestación de las competencias.

\section{Primeros pasos que relacionan la actitud con la competencia}

Una de las primeras aproximaciones para relacionar la actitud con la competencia es la de constatar cómo la literatura sobre la competencia se relaciona con la actitud. Dicha literatura se resume fundamentalmente en dos propuestas o acepciones, a saber: a) la que la actitud (especialmente, su dimensión afectiva) es sólo una parte de la competencia y, b) la que sugiere que la actitud y la competencia presentan algunos elementos semejantes puesto que ambas integran las tres dimensiones de la conducta humana y se evalúan en su ejecución, a partir de las evidencias de acción.

Cada una de estas acepciones presenta diferencias y similitudes en la manera de considerar las actitudes y las competencias. En la primera acepción, si en las definiciones de competencia se reduce la actitud a los valores o a las emociones en forma de "saber ser" o "saber estar", hecho que contradice el concepto triádico y actual de la mayor parte de la literatura sobre la actitud, se prescinde de las aportaciones de los otros dos factores de la actitud: los conceptos y la predisposición conductual, por lo que difícilmente este concepto sesgado de la actitud podrá ser útil. En la segunda acepción, en cambio, si se identifica, de forma paralela, el concepto de competencia con el de actitud, se clarifican algunos de los elementos relacionados con el origen, desarrollo y ejecución tanto de la actitud como de las competencias, al 
tiempo que se ofrecen pistas para comprender, interpretar e intervenir en la mejora de las competencias de acuerdo con las exigencias de futuro.

\section{$1^{a}$ acepción: la actitud es sólo una parte de la competencia}

Atendiendo a la definición integradora de actitud, resulta difícil de entender y justificar que la actitud sea sólo una parte de la competencia tal y como sucede en la mayoría de definiciones de competencia profesional consultadas (Pereda y Berrocal, 2001; Repetto, 2003; Repetto, Ballesteros y Malik, 2000; Repetto, Pena, Mudarra y Uribarri, 2007; Roe, 2003; Tejada, 2005; Tejada y Navío, 2004) y, en los recopilatorios realizados por Echeverría (2001) y Rodríguez Moreno (2006). Muchos de los autores citados se sitúan en un enfoque de competencia de naturaleza holística y compleja, definiéndola como un conjunto de conocimientos (saber), procedimientos (saber hacer) y actitudes (saber ser y saber estar) combinados, coordinados e integrados que, junto con la movilización de los recursos del entorno, capacitan a una persona para actuar con eficacia en situaciones profesionales diversas. En este sentido, la mayoría de autores, integran el término actitud en la definción de competencia pero lo identifican con una de sus dimensiones: la afectiva, en términos de "saber ser" y de "saber estar", obviando así la influencia de los factores conductuales y cognitivos en la presencia de la actitud. Reducir la actitud sólo a los contenidos afectivos contradice, o por lo menos disiente de la comprensión general del concepto de actitud que incluye las tres dimensiones ya mencionadas.

Por el contrario, si se admite que las emociones configuran la actitud juntamente con los otros dos factores, presentes como elementos personales de la competencia, nos encontraríamos en una situación, en parte similar a la ya descrita, que nos permitiría relacionar la competencia con la actitud. En este sentido, los elementos personales de la competencia serían los mismos que influyen en la formación y ejecución de la actitud (cognitivo, conductual y afectivo). Lo que sí parece evidente es que la actitud acompaña a la competencia, tanto en su origen como en su desarrollo y ejecución. El problema está en dilucidar hasta que punto se identifica y se diferencia de la misma competencia. 
$2^{a}$ acepción: aspectos similares que relacionan la actitud con la competencia

La actitud y la competencia presentan aspectos similares en su origen, desarrollo y manifestación. Tanto la actitud como la competencia se presentan como el resultado de incidir en las ideas, emociones y conductas. Es cierto que, probablemente, la actitud se presenta más como una tendencia y la competencia como una acción, pero de hecho, aunque provengan de perspectivas diferentes las dos son el resultado de la influencia de los tres componentes ya mencionados. Los diferentes niveles entre los componentes de la actitud y la competencia pueden explicar algunos de los matices existentes entre ellos:

- El hecho de poder incidir en los tres componentes: cognitivos, afectivos y conductuales en diferentes niveles y gradaciones, ofrece una gran variedad de posibilidades y matices que explican tanto el inicio, el desarrollo y la expresión de la actitud y de la competencia.

- Existe una larga tradición en considerar la preparación o formación de las competecias y su evaluación en situación real. Seria muy difícil entender la predisposición sin contemplar su ejecución y asimismo considerar la eficacia de la competencia sin integrar en la misma ejecución aspectos actitudinales.

- La distinción entre ser capaz y demostrarlo, potencia y acto, saber hacer y hacerlo, capacidades y competencias, preparación y ejecución, formación y aplicación, proceso y producto, inclinación y expresión de la actitud...etc. son conceptos que se integran perfectamente en el proceso de mejorar la información, los hábitos de conducta y las emociones, para preparar la ejecución de la actitud o la manifestación de la competencia. La distinción de algunos autores entre predisposición e inclinación a expresar la actitud (Eagly y Chaiken, 2007; Schwarz, 2007; Schwarz y Bohner, 2001) es, probablemente, similar al proceso en el que la información, los hábitos y las emociones configuran la competencia y su demostración, que permitirá constatar la posterior acreditación.

El hecho de que la actitud y la competencia compartan los tres elementos del comportamiento humano: cognitivo, afectivo y conductual, ofrece ciertas pistas para la comprensión de los procesos de confluencia entre ambos conceptos. También el enfoque del cambio de actitudes presenta dos perspectivas: una transversal, que incluye la incidencia de los tres fac- 
tores, y otra, centrada en el resultado final de la confluencia de dichos factores (Montané et al., 2007). La perspectiva evolutiva del resultado final de la actitud, nos introduce en una visión de proceso tanto de la competencia como de la actitud (Visser, Bizer, y Krosnick, 2006; Visser y Krosnick, 1998).

\section{La evaluación de la ejecución de la actitud y la competencia: un proceso de confluencia mutua.}

La evaluación tanto de la actitud como de la competencia, es la que nos indica su verdadero valor y es el punto dónde ambos conceptos se igualan. Es cierto que la actitud mira hacia la predisposición y la competencia hacia la resolución eficaz de la acción, pero una y otra adquieren valor de objetividad con la evaluación de su ejecución. Así pues, un punto importante de la competencia es la de valorarla en función de su ejecución (Echeverría, 2008; Rodríguez Moreno, 2006; Tejada 2005; Tejada y Navío 2004). En principio puede parecer que la actitud se fundamenta en la inclinación en ponerla en práctica, pero la actitud adquiere, también, valor en función de su aplicación (Visser et al., 2006).

Tanto la competencia como la actitud tienen como prioritaria la evaluación de su ejecución o manifestación, si bien la actitud se entiende como predisposición y la competencia como acción. Para la actitud, la evaluación de su ejecución es un indicador que permite constatar hasta que punto la predisposición a actuar se evidencia. La investigación evaluativa permite constatar hasta que punto la actitud explica un porcentaje determinado de su realización y por extensión, de la ejecución de la competencia.

Asimismo la amplia literatura relacionada con la competencia coincide en afirmar que ésta sólo adquiere valor si se demuestra en la acción. La convergencia entre la actitud y la competencia habrá que buscarla pues en que la actitud y la competencia adquieren valor y se asimilan cuando se ejecutan; el final de la actitud es su manifestación en forma de comportamiento, hábito o automatismo mientras que el de la competencia es la eficacia de su ejecución. La evaluación de actitudes y competencias son dos formas de constatar el resultado de la formación y garantizar, a través de procedimientos de transferencia, la motivación para la ejecución y resolución eficaz de las tareas profesionales que se derivan de un puesto de traba- 
jo. En este sentido, la competencia necesita de la actitud si bien ésta no parece ser suficiente para una ejecución competente.

Probablemente la diferencia entre actitud y competencia está en que la actitud mira más la predisposición a actuar mientras que la competencia se centra en la misma ejecución del comportamiento.

\section{La actitud y la competencia se modifican con la ejecución}

La manifestación de la actitud, y probablemente la de la competencia, así como su evaluación en la acción, influyen a su vez en los mismos elementos que les ayudaron a emerger. Este aspecto explica como la ejecución se transforma en rutinas o en comportamientos y como éstos pueden remodelar de nuevo las ideas, las destrezas y hábitos e incidir en las emociones, llegando a generar nuevas actitudes que impliquen o predispongan nuevos comportamientos y que ayuden, a través del aprendizaje en la acción, a la adquisición y desarrollo de nuevas competencias.

Tanto la manifestación de la actitud como de la competencia implica un conjunto de evaluaciones que a su vez implican modificaciones positivas o negativas que se podrían explicar según la forma y evolución en la ejecución. Los conceptos de adaptación al cambio, la transferencia de los aprendizajes, el aprendizaje creativo, etc., son maneras de explicar cómo la ejecución de la actitud y la competencia explican, en parte, su evolución y desarrollo. También la misma ejecución de la actitud y la competencia influyen en los elementos cognitivos, afectivos y conductuales que las desarrollaron.

A modo de conclusión, de todo lo dicho podría parecer que la actitud, por su carácter de predisposición, finaliza con la ejecución y que, por su parte, la competencia se demuestra y se perfecciona con su posterior ejecución. Pero la actitud acompaña a la competencia en todo el proceso de adquisición, ejecución y evaluación y se configura con los mismos tres elementos que influyen en las competencias: cognitivo, afectivo y conductual. Este mismo motivo sugiere que, por su estructura y génesis análoga, los componentes de la actitud y de la competencia presentan características bastante similares. Si bien parece cierto que hay acuerdo en presentar los tres componentes ya citados: cognitivo, afectivo y conductual como los factores que explican el origen y evolución tanto de las actitudes como de las competencias, 
habrá que dilucidar cuales son las diferencias específicas o formales entre las actitudes y las competencias. Probablemente, al menos en parte, habrá que buscarlas en la estructura triádica de los tres componentes y en su interrelación. Si la competencia se explica en parte, por su origen, desarrollo y evaluación de la ejecución de manera similar a las actitudes, éstas probablemente, también la acompañan en su posterior evolución. Por consiguiente, habrá que aceptar que entre actitudes y competencias existen conexiones fundamentales relacionadas con su origen, desarrollo y ejecución y que dichas conexiones van más allá de una simple relación con el factor afectivo de la actitud.

\section{Algunas implicaciones educativas desde las actitudes y competencias}

El modelo cambio de actitudes de Montané et al. (2007), aplicado a la reducción de accidentes de tráfico, no se limita a la predisposición a actuar, sino que contempla la misma ejecución de la actitud con el propósito de practicar comportamientos seguros relacionados con la conducción eficaz y responsable. Por lo que probablemente, algunas de las manifestaciones están más próximas a la competencia que a la ejecución de la actitud.

El modelo de cambio de actitudes integra una secuencia de cinco fases (evaluación inicial, mejora de la información, revisión de hábitos de comportamiento, integración de emociones y evaluación final) y fundamenta el diseño, aplicación y evaluación de diferentes programas de educación vial, elaborados por la Cátedra de Educación y Formación Vial de la Universidad Autónoma de Barcelona y el Servei Català de Trànsit, que se aplican en la formación de preconductores, conductores, conductores profesionales y conductores reincidentes de todo Cataluña: programa de seguridad vial a preconductores, programa de conducción segura para motos de gran cilindrada, programa de conducción segura para conductores profesionales de autobús y camiones, programa de desarrollo global desde la conducción segura para usuarios internos de prisiones y programa taller de conducción segura para conductores reincidentes, todos con el objetivo de reducir el comportamiento de riesgo en la conducción.

La aplicación y evaluación de los resultados de dichos programas, fundamentados en el cambio de actitudes, presentan resultados significativos desde el punto de vista de la intención y ejecución del comportamiento seguro y eficaz en la conducción, puesto que no sólo se considera la actitud como la predisposición a actuar sino también como la ejecución real del 
comportamiento manifestado en la conducción, por lo que la actitud adquiere valor cuando se transforma en comportamiento esperado y/o en competencia. En este caso, la actitud presentada como predisposición a actuar se transforma en ejecución real o demostración, hecho muy similar a la misma definición de competencia, especialmente si se tiene en cuenta la eficacia en la resolución de la acción.

Los siguientes casos son ejemplo de lo dicho anteriormente. Los resultados obtenidos en una muestra de 134 preconductores, aplicando el programa de seguridad vial a preconductores, mejoran su nivel de seguridad en 15 puntos sobre 50 en relación al grupo control con una significación de $p<.001$ (Jariot y Montané, 2009). Asimismo el Programa de Educación del Conductor (PEC), aplicado a una muestra de 24 conductores temerarios, éstos mejoran en un $75 \%$ en los ítems relacionados con las actitudes de riesgo en la conducción, tratados en la aplicación del programa; además, estos resultados permanecen estables después de 6 meses, manifestando la ejecución de comportamientos seguros y eficaces en la conducción (Montané y Ferrer, 1993). También el programa taller de conducción segura, aplicado a una muestra de 54 usuarios de medidas penales alternativas al cabo de 6 meses, un $86 \%$ de los encuestados recuerda los compromisos de seguridad en la conducción realizados al término del programa y manifiestan, en menor proporción (75\%), sus esfuerzos en mantener dichos compromisos y en llevarlos a la práctica a través de comportamientos de movilidad segura. Todos estos hechos, probablemente, son el resultado de traspasar la frontera de la actitud a la competencia, o de la predisposición a actuar hasta la ejecución real y eficaz del comportamiento.

En conclusión los programas fundamentados en el modelo de cambio de actitudes presentan resultados satisfactorios, probablemente, también, porque dichos programas, aunque se fundamenten en este modelo, incluyen un alto componente de ejecución práctica con muchas semejanzas con las competencias.

\section{Referencias}

Ajzen, I. (2001). Nature and Operation of Attitudes. Annual Review of Psychology, 58, 27-52. Ajzen, I. y Fishbein, M. (1980). Understanding attitudes and predicting social behaviour. Englewood Cliffs, NJ: Prentice-Hall. 
Ajzen, I. y Fishbein, M. (2005). The Influence of Attitudes on Behavior. En D. Albarracín, B.T. Johnson, y M.P. Zanna (Eds.), The Handbook of Attitudes (pp.173-222). Mahwah, NJ: Lawrence Erlbaum Associates, Inc.

Albarracín, D., Johnson, B.T., Zanna, M.P. y Kumkale, G.T. (2005). Attitudes: Introduction and Scope. En D. Albarracín, B.T. Johnson, y M.P. Zanna (Eds.), The Handbook of Attitudes (pp.3-20). Mahwah, NJ: Lawrence Erlbaum Associates, Inc.

Allport, G. W. (1935). Attitudes. In C. Murchison (Ed.), Handbook of social psychology (pp. 798-844). Worchester, MA: Clark University Press.

Armitage, C. J. y Conner, M. (2000). Attitudinal ambivalence: A test of three key hypotheses. Personality and Social Psychology Bulletin, 26, 1421-1432.

Arnau, L (2010). La orientación de las personas desempleadas para la adaptación al cambio profesional: elaboración, aplación y evaluación de un programa de orientación aplicado a alumnos de formación professional para el empleo. Tesis Doctoral no publicada. Barcelona (Bellaterra): Universidad Autónoma de Barcelona.

Arnau, L. y Montané, J. (2010). Indicadores para mejorar el desarrollo profesional de los aspirantes a profesores de formación vial en Cataluña. Revista Española de Orientación y Psicopedagogía, 21(2), 295-307.

Aronson, E. (1968). Dissonance theory: progress and problems. En R.P. Abelson, E.Aronson, W.J. McGuire, T.M. Newcomb, M.J. Rosenberg y P.H. Tannenbaum (Eds.), Cognitive consistency theories: a source book (pp. 5-27). Skokie, IL: Rand McNally.

Aronson, E. (1999) Dissonance, hypocrisy, and the self-concept. En E. Harmon-Jones y J. Mills (Ed.), Cognitive dissonance: progress on a pivotal theory in social psychology (pp. 103-126). Washington, DC: American Psychological Association.

Bohner, G. y Wänke, M. (2002). Attitudes and attitude Change. New York, NY: Psychology Press.

Clore, G. L. y Schnall, S. (2005). The Influences of Affect on Attitude. En D. Albarracín, B. T. Johnson, y M. P. Zanna (Eds.), The Handbook of Attitudes (pp.437-493). Mahwah, NJ: Lawrence Erlbaum Associates, Inc

Cooper, J. y Fazio, R.H. (1984) A new look at dissonance theory. En L. Berkowitz (Ed.), Advances in experimental social psychology (pp. 229-266). San Diego, CA: Academic Press.

Eagly, A.H. y Chaiken, S. (1993). The Psychology of Attitudes. Fort Worth, TX: Harcourt Brace. 
Eagly, A.H. y Chaiken, S. (1998). Attitude structure and function. En D. Gilbert, S. Fiske, y G. Lindzey (Eds.), The Handbook of Social Psychology (4rt ed., pp.269-322). New York: McGraw-Hill.

Eagly, A. y Chaiken, S. (2005). Attitude Research in the $21^{\text {st }}$ Century: The Current State of Knowledge. En D. Albarracín, B.T. Johnson, y M.P. Zanna (Eds.), The Handbook of Attitudes (pp.743-768). Mahwah, NJ: Lawrence Erlbaum Associates, Inc.

Eagly, A. y Chaiken, S. (2007). The advantages of an inclusive definition of attitude. Social Cognition, 25, 582-602.

Echeverría, B. (2001). Configuración actual de la profesionalidad. Letras de Deusto, 31 (91), $35-56$.

Echeverría, B. (Coord.) (2008). Orientación Profesional. Barcelona: UOC

Erwin, P. (2001). Attitudes and persuasion. New York: Psychology Press.

European Communities. (2008). Qualifications Framework for Lifelong Learning. Luxembourg: Office for Official Publications of the European Communities.

Fabrigar, L.R., MacDonald, T.K. y Wegener, D.T. (2005). The Structure of Attitudes. En D. Albarracín, B.T. Johnson, y M.P. Zanna (Eds.), The Handbook of Attitudes (pp.79124). Mahwah, NJ: Lawrence Erlbaum Associates, Inc.

Fazio, R.H. y Zanna, M.P. (1981). Direct experience and attitude behavior consistency. En L. Berkowitz (Ed.), Advances in experimental social psychology (pp.161-202). New York: Academic Press

Fazio, R.H. (1995). Attitudes as object-evaluation associations: determinants, consequences, and correlates of attitude accessibility. En R.E. Petty, y J.A. Krosnick (Eds.), Attitude strength: antecedents and consequences (pp.247-282). Hillsdale, NJ: Erlbaum

Fazio, R.H. y Olson, M.A. (2003). Implicit Measures in Social Cognition Research: Their Meaning and Use. Annual Review of Psychology, 54, 297-327.

Festinger, L. (1957). A theory of cognitive dissonance. Stanford, CA: Stanford University Press.

Gawronski, B. (2007). Attitudes can be mesured! But what is an actitude? Social Cognition, $25,573-581$.

Gawronski, B. y LeBel, E.P. (2008). Understanding patterns of attitude change: When implicit measures show change, but explicit measures do not. Journal of Experimental Social Psychology, 44, 1355-1361.

Gimeno Sacristán, J (2008). Educar por competencias ¿Que hay de nuevo?. Madrid: Morata 
Haddock, G. y Maio, G. (2004). Contemporary perspectives on the psychology of attitudes. Philadelphia: Psychology Press.

Heider, F. (1946). Attitudes and cognitive organization. Journal of Psychology, 21, 107-112.

Heider, F. (1958). The psychology of interpersonal relations. New York: Wiley

Holland, R.W., Verplanken, B. y Knippenberg, A. (2002). On the nature of attitude-behavior relations: the strong guide, the weak follow. European Journal of Social Psychology, $32,869-876$.

Jariot, M. y Rodríguez Parrón, M. (2007). La formación por competencias profesionales. Evaluación y mejora de las competencias del profesor de formación vial desde un modelo de cambio de actitudes. Educación XXI, 10, 107-136

Jariot, M. y Montané, J. (2009). Actitudes y velocidad en jóvenes. Aplicación de un programa de educación vial. Relieve, 15(1).

Johnson, B.T., Maio, G.R. y Smith-McLallen, A. (2005). Communication and Attitue Change: Causes, Process and Effects. En D. Albarracín, B.T. Johnson, y M.P. Zanna (Eds.), The Handbook of Attitudes (pp.617-670). Mahwah, NJ: Lawrence Erlbaum Associates, Inc.

Krosnick, J.A., Judd, C.M. y Wittenbrink, B. (2005). The Measurement of Attitudes. En D. Albarracín, B.T. Johnson, y M.P. Zanna (Eds.), The Handbook of Attitudes (pp.21-77). Mahwah, NJ: Lawrence Erlbaum Associates, Inc.

Kruglanski, A.W. y Stroebe, W. (2005). The influence of beliefs and goals on attitudes: Issues of structure, function and dynamics. En D. Albarracín, B.T. Johnson, y M.P. Zanna (Eds.), The Handbook of Attitudes (pp.323-369). Mahwah, NJ: Lawrence Erlbaum Associates, Inc.

Levitan, L. C. y Visser, P. S. (2008). The impact of the social context on resistance to persuasion: Effortful versus effortless responses to counter-attitudinal information. Journal of Experimental Social Psychology, 44(3), 640-649

Ley Orgánica de las Cualificaciones y de la Formación Profesional (2002, 19 de junio). Boletín Oficial del Estado, junio 20, 2002.

Maio, G. y Haddock, G. (2010). The psychology of attitudes and attitude change. London, UK: SAGE Publications Ltd.

Marsh, K.L. y Wallace, H.M. (2005). The influence of attitudes on beliefs: Formation and change. En D. Albarracín, B.T. Johnson, y M.P. Zanna (Eds.), The Handbook of Attitudes (pp.369-397). Mahwah, NJ: Lawrence Erlbaum Associates, Inc. 
Montané, J. (1982). La teoría de la Consistencia Disonancia versus la teoría de la autoestima. Una nueva perspectiva para futuras investigaciones. Revista de Psicología General y Aplicada, 37, 861-876.

Montané, J. (1983). La teoría de la Consistencia Disonancia versus la teoría de la autoestima. Una nueva perspectiva para futuras investigaciones. Revista de Psicología General y Aplicada, 38, 467-479.

Montané, J. y Ferrer, F. (1993). Cuaderno de formación de formadores del conductor: Programa de educación del conductor. Barcelona: PPU.

Montané, J., Martínez, M. y Jariot, M. (2004). La pedagogia aplicada a una conducció segura i eficaç. Barcelona: Generalitat de Catalunya. Departament d'Interior. Servei Català de trànsit.

Montané, J. y Jariot, M. (2005). La psicologia aplicada a una conducció segura i eficaç. Barcelona: Generalitat de Catalunya. Departament d'Interior. Servei Català de trànsit.

Montané, J., Jariot, M. y Rodríguez, M. (2007). Actitudes, Cambio de Actitudes y Conducción segura: Un enfoque crítico aplicado a la reducción de accidentes. Barcelona: Laertes.

Montané, J. (2010). Programas de educación vial, fundamentados en el cambio de actitudes, para la movilidad segura. En C. Jimenez (Coord.), Educación y seguridad vial. Madrid: Ed. Davinci.

Olson, J.M. y Stone, J. (2005). The Influence of Behaviour on Attitudes. En D. Albarracín, B.T. Johnson, y M.P. Zanna (Eds.), The Handbook of Attitudes (pp.223-271). Mahwah, NJ: Lawrence Erlbaum Associates, Inc.

Pereda, S. y Berrocal, F. (2001). Técnicas de gestión de recursos humanos por competencias. Madrid: Editorial Centro de Estudios Ramón Areces.

Pérez Gómez, A. (2008). ¿Competencias o pensamiento práctico? La construcción de los significados de representación y de acción. En J. Gimeno Sacristán (Comp.), Educar por competencias ¿Que hay de nuevo? (pp. 59-103). Madrid: Morata

Petersen, K. R. y Dutton, J. E. (1975). Centrality, extremity, intensity: Neglected variables in research on attitude-behavior consistency. Social Forces, 54, 393-414.

Repetto, E., Ballesteros, B. y Malik, B. (2000). Tareas y formación de los orientadores en la Unión Europea. Madrid: UNED

Repetto, E. (2003). La competencia emocional e intervenciones para su desarrollo. En E. Repetto (Ed.), Modelos de Orientación e Intervención Psicopedagógica (pp. 453-482.). Madrid: UNED. 
Repetto, E., Pena M., Mudarra, M.J. y Uribarri, M. (2007). Orientación de las competencias socioemocionales de los alumnos de Educación Secundaria en contextos multiculturales. Electronic Journal of Research in educational Psychology, 11(5), 159-178.

Roe, R. (2003). What makes a competent psychologist? European Psychologist, 7(3), 192202.

Rodríguez Moreno, M.L. (2006). Evaluación, balance y formación de competencias laborales transversales: Propuestas para mejorar la calidad en la formación profesional y en el mundo del trabajo. Barcelona: Laertes.

Schwarz, N. y Bohner, G. (2001). The construction of attitudes. En A. Tessery y N.Schwarz (Eds.), Blackwell handbook of social psychology: Intraindividual processes (pp. 436457). Malden, MA: Blackwell Publishers.

Schwarz, N. (2007). Attitude construction: Evaluation in context. Social Cognition, 25(5), 638-656.

Tejada, J. (1999a). Acerca de las competencias profesionales I. Herramientas, 56, 20-30.

Tejada, J. (1999b). Acerca de las competencias profesionales II. Herramientas, 57, 8-14.

Tejada, J. (2005). El trabajo por competencias en el prácticum: cómo organizarlo y cómo evaluarlo. Revista Electrónica de Investigación Educativa, 7(2).

Tejada, J. y Navío, A. (2004). El desarrollo y la gestión de las competencias profesionales: una mirada desde la formación. Revista Iberoamericana de Educación, 37(2), 1-15.

Thurstone, L.L. (1931). Attitudes can be measured. American Journal of Sociology, 33, 529554 .

Visser, P. S. y Krosnick, J. A. (1998). The development of attitude strength over the life cycle: Surge and decline. Journal of Personality and Social Psychology, 75, 1389-1410.

Visser, P. S., Bizer, G. y Krosnick, J. A. (2006). Exploring the latent structure of strengthrelated attitude attributes. En M. Zanna (Ed.), of Advances in Experimental Social Psychology, Volume 38 (pp. 1-67). San Diego, CA: Academic Press.Visser, P. S., Bizer,

Zanna, M.P. y Rempel, J.K. (1988). Attitudes: A new look at an old concept. En D. Bar-Tal y A.W. Kruglanski (Eds.), The social psychology of knowledge (pp.315-334). Cambridge, UK: Cambridge University Press. 